\title{
Threshold and conversion experiments on seeded stimulated Raman scattering
}

\author{
R.J. Heeman ${ }^{1}$, H.P. Godfried ${ }^{2}$, W.J. Witteman ${ }^{1}$ \\ ${ }^{1}$ University of Twente, Department of Applied Physics, P.O. Box 217, 7500 AE Enschede, The Netherlands \\ (Fax: + 31-53/338065) \\ 2 Nederlands Centrum voor Laser Research (NCLR), P.O. Box 2662, 7500 CR Enschede, The Netherlands \\ (Fax: + 31-53/338065)
}

Received: 5 July 1994/Accepted: 18 November 1994

\begin{abstract}
High energy-conversion efficiencies in Stimulated Raman Scattering (SRS) are demonstrated both in experiments and by simulations for pump powers below SRS threshold. The scattering is induced by a short seed pulse at the Stokes frequency, the pulse width of which is much shorter than the pump pulse width and which is comparable with the medium's dephasing time.
\end{abstract}

PACS: $42.50 . \mathrm{Md} ;$ 42.55.Ye; 42.65.Dr

In a Raman-active medium, high conversion efficiencies to the Stokes wave can be achieved by Stimulated Raman Scattering (SRS). Due to the low gain for scattering to Stokes waves in the infrared, high pump powers and long interaction paths are necessary. Additionally, when the scattering becomes transient for pump pulses with a pulse duration comparable to the medium's dephasing time, the Raman gain is further reduced. One method to overcome the low Raman gain is by seeding. The scattering is then made efficient by an initially applied Stokes field rather than started up from the Stokes-noise freld. The seed power easily exceeds the Stokes-noise power by many orders of magnitude, and substantially lowers the gain necessary to obtain Stokes powers comparable to the pump power. In previously performed experiments [1], the conversion efficiency in transient scattering was increased by a seed pulse with a pulse duration comparable to that of the pump pulse.

\section{Experiments}

In the experiments described in this paper, the SRS efficiency of a pump pulse in the infrared is increased by adding a seed pulse at the Stokes frequency which is much shorter than the pump pulse, while the medium's dephasing time is of the same order of magnitude as the seed pulse. It is demonstrated both in experiments and in simulations that this short seed pulse is able to induce SRS with high energy-conversion efficiency, where unseeded scattering would otherwise generate Stokes energies below detection levels.

In these experiments, a $10.26 \mu \mathrm{m}$ pump pulse from a TEA $\mathrm{CO}_{2}$ laser with a pulse width of $70 \mathrm{~ns}$ at half maximum and a pump energy in the gain-switched peak of maximum $1.4 \mathrm{~J}$ was scattered by SRS in para-hydrogen $\left(\mathrm{p}-\mathrm{H}_{2}\right)$. The pump beam was nearly TEM $_{00}$ but the pulse shape generally showed small modulation due to mode beating. The $S_{0}(0)$ rotational transition was used as the Raman transition. For this transition, the pressure dependence of the dephasing time $T_{2}$ of the Raman polarizability can be expressed as $T_{2}=(\pi B p)^{-1}$ with $B=97.3 \mathrm{MHz} /$ bar [2] for pressures $p$, where the Raman line width is pressure broadened, i.e., for pressures above approximately 0.3 bar [3]. The Raman shift of $354 \mathrm{~cm}^{-1}$ shifts the pump wavelength to a Stokes wavelength of $16.11 \mu \mathrm{m}$. To increase the small Raman gain at these long Stokes wavelengths, a Multi-Pass Cell (MPC) [4] was used. The MPC consisted of two spherical mirrors separated by $3.7 \mathrm{~m}$ in a near-concentrical, stable-cavity setup in which the pump and Stokes beams were focused at each pass with a confocal parameter of $72 \mathrm{~cm}$. In the $\mathrm{MPC}$, the number of passes could be varied with an axially mounted rotatable periscope which could reflect the beam out of the MPC after an even number of passes between 2 and 32. The powers available from the $\mathrm{CO}_{2}$ laser were so low that no scattered radiation in the first Stokes wave was detectable unless the scattering was seeded by a lowpower seed at the Stokes frequency. In the seeded experiments, higher-order Stokes waves were not generated and, consequently, using also a circularly polarized pump beam, no anti-Stokes waves were generated since the circular polarization excludes Stokes-anti-Stokes coupling. The seed pulse at the Stokes-shifted frequency of the $\mathrm{CO}_{2}$ laser was generated in the same MPC by a Four-Wave Mixing (FWM) process, where the $1.064 \mu \mathrm{m}$ fundamental of a $Q$-switched Nd:YAG laser and its Stokes-shifted frequency were mixed with the $10 \mu \mathrm{m}$ pump 
wave [4]. Since the Stokes-shifted wavelength of the $\mathrm{Nd}$ :YAG pump is much shorter than the Stokes-shifted wavelength of the $\mathrm{CO}_{2}$ pump wave, the Stokes gain is approximately a factor 200 higher for scattering at $1 \mu \mathrm{m}$ [4]. Therefore, the scattering of the Nd:YAG pump wave resulted in the generation of many higher-order Stokes waves and, due to parametrical coupling, also anti-Stokes waves. First Stokes generation at $1.1 \mu \mathrm{m}$ occurred with $40 \%$ energy efficiency [4]. Using the conversion function for FWM derived by Bjorklund [5], a maximum seed power of approximately $5 \times 10^{-2} \mathrm{MW}$ may be estimated for the pump powers used in the experiments. This seed power is many orders of magnitude above the Stokespower level generated by spontaneous Raman scattering (of order $10^{-11} \mathrm{~W}$ ) from which the stimulated scattering of the $\mathrm{CO}_{2}$ pump wave starts. The seed pulse generated by FWM is then as short as the shortest of the three input waves. In these experiments, the seed-pulse width was determined by the 9 ns width of the Stokes-shifted wave of the Nd:YAG laser. In all experiments, the polarization of both pump beams was circular to maximize the Raman gain [6]. The pump beams were mode-matched to the MPC and collinearly injected into the MPC. The output beams were dispersed in a prism and directed to detectors to measure simultaneously the pulse energy (by means of pyroelectric detectors) and the pulse shapes (by means of photon-drag detectors and PIN photodiodes). A complete description of the experimental setup is given elsewhere [3].

In the next section, the results of the threshold experiments are given, while the experimental and numerical results of conversion in seeded stimulated Raman scattering are discussed in Sects. 3 and 4, respectively.

\section{Results of the threshold experiments}

In the following, the terms pump and Stokes refer to waves with the pump frequency and the Stokes-shifted frequency of the $\mathrm{CO}_{2}$ laser, respectively. Also, threshold refers to the SRS threshold of the $\mathrm{CO}_{2}$ pump wave unless the Nd:YAG pump wave is mentioned explicitly. The threshold condition was met when a Stokes pulse could be detected by the photon-drag detector. Then, the energy in the Stokes pulse was approximately $10 \mathrm{~mJ}$. The delay between the two pump pulses was adjusted to generate maximum Stokes power. In general, the peak of the $\mathrm{CO}_{2}$ pump pulse then coincided with or closely followed (a few ns) the peak of the Nd:YAG pump pulse.

The behavior of the threshold power is experimentally investigated as a function of Nd:YAG pump power, gas pressure and number of passes in the Raman cell. To be able to show the effects of seeding on the threshold power, the threshold power for unseeded SRS was first calculated from Nd:YAG threshold-power measurements, which were performed in the same Raman MPC. In these calculations, the gain was corrected for the difference in wavelength and for the difference in pulse width. For the latter, it was necessary to take into account the gain reduction due to transient scattering, which depends on the ratio of pulse width and dephasing time. The used gain-reduction factor was measured in previous experi-

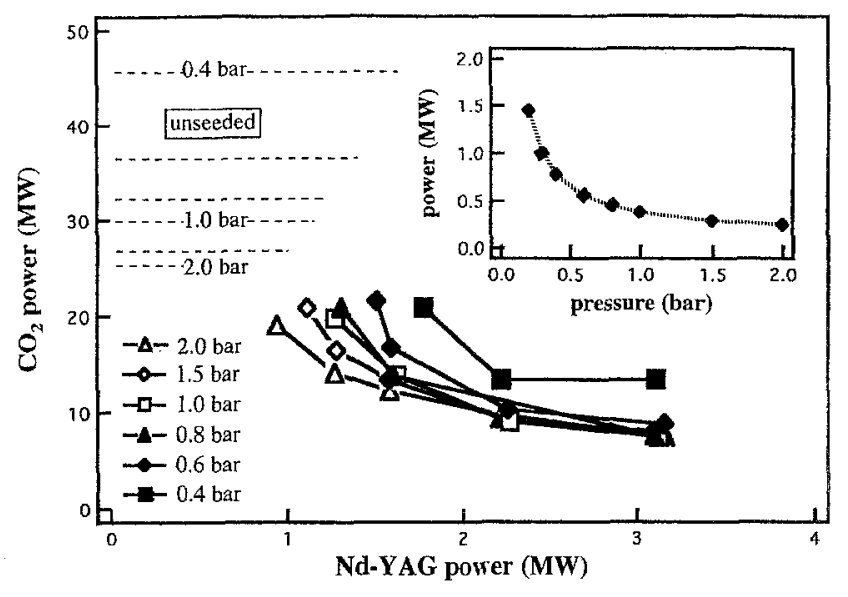

Fig. 1. Measured threshold powers (solid lines) in seeded SRS after 32 passes and calculated threshold powers (dashed lines) in nonseeded SRS as a function of Nd:YAG pump power at pressures ranging from 0.4 to 2 bar. The insert shows the Nd:YAG threshold power as a function of pressure

ments [3]. Typically, for unseeded SRS, a threshold power of $31 \mathrm{MW}$ is calculated for the $70 \mathrm{~ns} \mathrm{CO}_{2}$ pulse which propagates 32 passes through the MPC filled with $1.0 \mathrm{bar}$ $\mathrm{p}-\mathrm{H}_{2}$ at room temperature.

In Fig. 1, the measured seeded and calculated unseeded threshold powers are shown as a function of Nd:YAG pump power for several pressures. The insert shows the threshold power for pure SRS of the Nd:YAG pump pulse as a function of pressure, which was measured previously [3]. It is noted that the threshold data for seeded SRS of the $\mathrm{CO}_{2}$ pump pulse were obtained for Nd:YAG powers above SRS threshold for the Nd:YAG pump. For higher Nd:YAG pump powers and at gas pressures above $0.6 \mathrm{bar}$, the threshold power approaches approximately $6 \mathrm{MW}$, which is to be compared to the threshold of a few tens of MW necessary in unseeded SRS. At lower gas pressures, where the medium's dephasing time becomes long, transient scattering results in a low Raman gain for both the Nd:YAG and $\mathrm{CO}_{2}$ pump pulses. Therefore, the threshold powers for pure SRS of both pump pulses increase and the Stokes powers scattered from both pump waves decrease. This results in a low seed power generated by FWM and a low Stokes power generated in the seeded-scattering processes and causes the threshold-power curves at lower pressures to shift to higher Nd:YAG powers.

Figure 2 shows the threshold power as a function of gas pressure. The upper curve represents the calculated threshold power for unseeded SRS while the lower two curves represent the measured threshold power for seeded SRS at two Nd:YAG power levels of 3.2 and $1.6 \mathrm{MW}$. Above pressures where Nd:YAG-pumped SRS exceeds threshold ( 0.2 bar for $3.2 \mathrm{MW}$ and 0.3 bar for $1.6 \mathrm{MW}$ ), the threshold power is reduced. At higher pressures the threshold power approaches approximately 6 and $10 \mathrm{MW}$ for 3.2 and 1.6 MW Nd:YAG pump powers, respectively. The ratio of threshold powers in unseeded and seeded SRS is largest for gas pressures around 0.5 bar, since the unseeded SRS threshold power also decreases when the pressure is increased. 


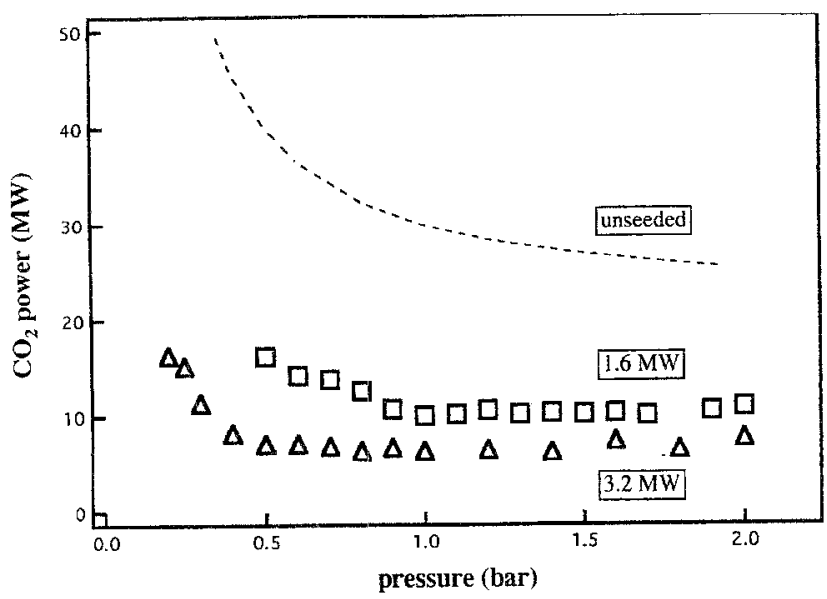

Fig. 2. Measured threshold powers in seeded SRS after 32 passes for a 1.6 and $3.2 \mathrm{MW}$ Nd:YAG pump power and calculated threshold powers in unseeded SRS as a function of pressure

Figure 3 shows the threshold power as a function of the number of passes through the MPC for several $\mathrm{Nd}: Y A G$ pump powers. In the range of number of passes examined, the measured threshold power shows only small curvature. In an extrapolation towards lower number of passes, the seed power generated by FWM is possibly comparable to the Stokes-noise power generated by spontaneous Raman scattering and, as a consequence, the threshold power should approach the unseeded threshold power.

In summary, the most significant result of seeding is the large reduction, up to a factor 5 , of the threshold power at gas pressures around 0.5 bar.

\section{Results of the conversion experiments}

In the seeded SRS experiments, the scattering of $\mathrm{CO}_{2}$ radiation to the Stokes-shifted frequency depends on many parameters. Among these, the power of both pump lasers, the delay between the pulses, the total interaction length or the number of passes in the Raman cell, and the $\mathrm{p}-\mathrm{H}_{2}$ gas pressure are varied. Both pump powers determine the seed power generated by FWM, while the $\mathrm{CO}_{2}$ pump power additionally determines the Stokes amplification by SRS. The energy of the generated Stokes pulse sensitively depends on the delay between the two pump pulses. When the pump pulses overlap at the peaks, a maximum seed power is generated. However, as the experiments show, this condition not necessarily results in the highest Stokes energy.

To study experimentally the behavior of the pump and Stokes pulse shapes in seeded SRS, first, the delay was varied to find a value for which maximum Stokes energy was generated. This delay and the corresponding Stokes energy and pulse width were measured as a function of the number of passes, pump powers and gas pressure. These three quantities-delay, energy, pulse width-are presented in graphs where each graph is accompanied by a series of measured and calculated input and output pulse shapes. In this section, the experimental results are presented, while in the next section the mechanism of seeded SRS is

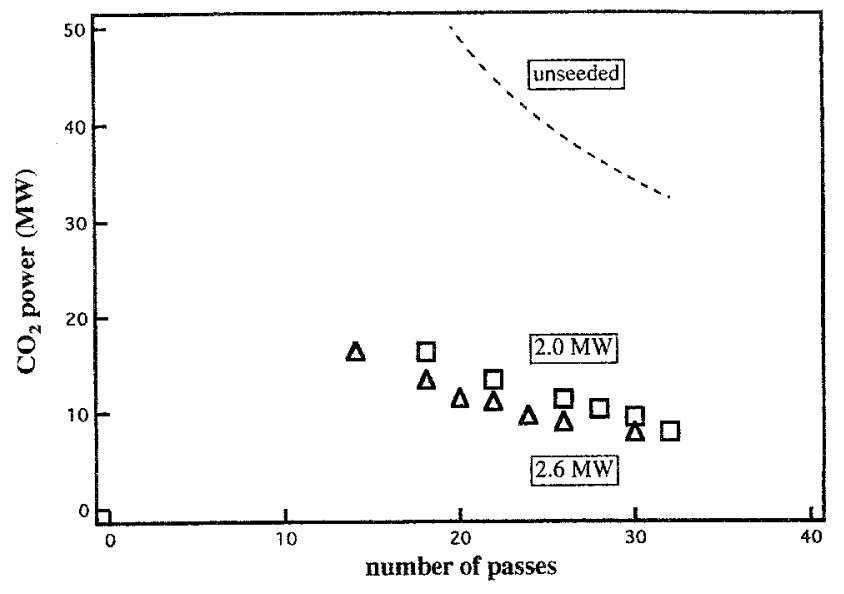

Fig. 3. Measured threshold powers in seeded SRS for a 2.0 and 2.6 MW Nd:YAG pump power and calculated threshold powers in unseeded SRS as a function of number of passes

discussed, using the concept of a polarizability with a finite dephasing time, and the experimental pulse shapes are compared with the calculated pulse shapes.

Figure 4 shows the energy, width and peak power of the generated Stokes pulses as a function of the delay. The pump power was taken high enough to cause full depletion of the $\mathrm{CO}_{2}$ pump pulse for optimum timing. The delay is taken as positive when the $\mathrm{CO}_{2}$ pump pulse leads the Nd:YAG pump pulse, and vice versa, as negative; at zero delay, the peaks of both pulses coincide. Maximum energy conversion is obtained when the Nd:YAG pump pulse leads the $\mathrm{CO}_{2}$ pump pulse by a few tens of ns. Then, a Stokes pulse is generated with the largest width and the pump pulse is fully and symmetrically depleted. For negative values of the delay, the Stokes pulse is generated just after or at the peak of the pump pulse. For positive values the Nd:YAG pump pulse determines the position of the Stokes pulse, which generally contains little energy.

In Fig. 5, the number of passes in the multi-pass Raman cell is varied. For high number of passes, when full pump depletion is obtained, the Stokes peak power and the delay cease to increase, whereas the Stokes energy continues to increase. This is due to the increasing Stokespulse width, which is partly determined by the delay and partly by the number of passes. The delay has to be set to more negative values to obtain the highest Stokes energy, while at higher number of passes, the pump pulse is also depleted further in the tail. A smaller delay is necessary for smaller number of passes where the Stokes power approaches the threshold level.

Figure 6 shows the dependence on the pump power. For low pump powers, where the pump pulse is not depleted, the Stokes power is growing nonlinearly. When depletion sets in, the growth of the Stokes power changes to linear with a slope equal to the product of the quantum efficiency and the cell transmission. This is represented by the straight line in Fig. 6. For maximum energy conversion at higher pump powers, the delay of the $\mathrm{CO}_{2}$ pump pulse relative to the Nd:YAG pump pulse was increased slightly to allow a wider pump depletion and Stokes pulse width. In addition, the pump pulse was depleted further in the tail. The maximum delay between the pump pulses at 


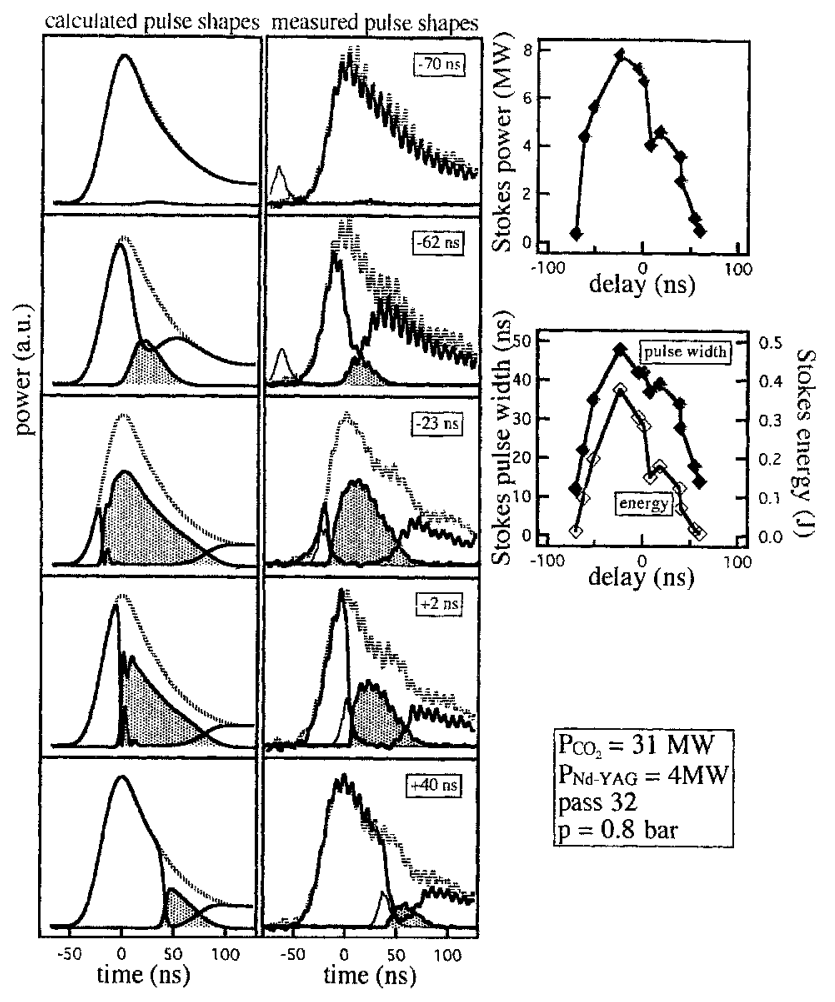

Fig. 4. Stokes energy, pulse width, and power in seeded SRS as a function of the delay between the two pump pulses. A negative delay represents a leading Nd:YAG pump pulse. Grey line: input pump pulse; thick lines: output pump and Stokes pulses where the surface below the Stokes pulse is shaded; thin line: $\mathrm{Nd}$ :YAG input pulse
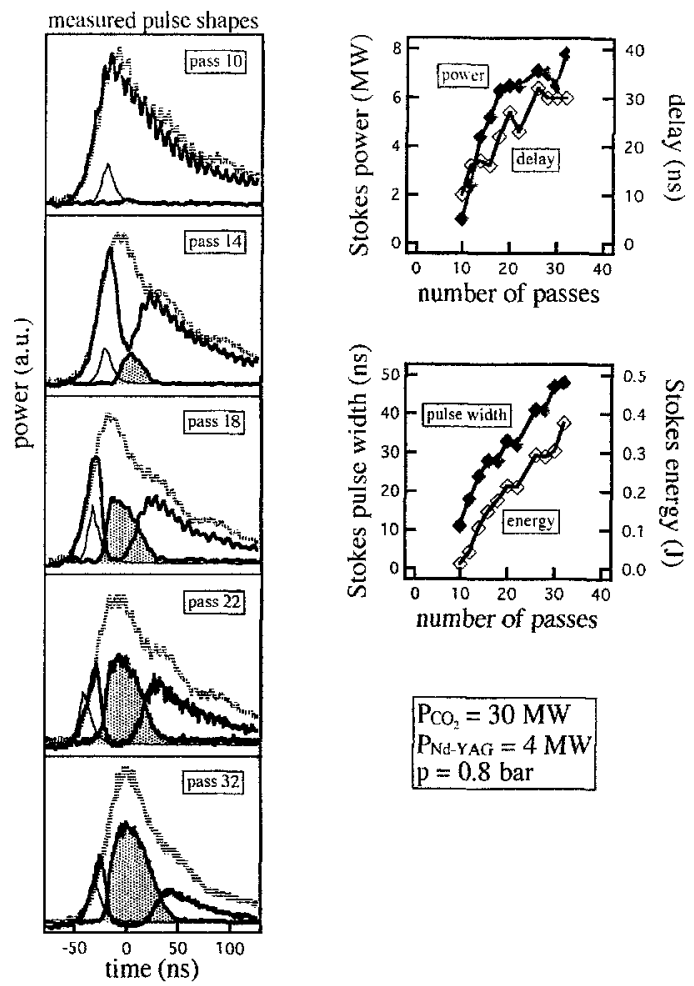

Fig. 5. Stokes energy, pulse width, power, and the delay in seeded SRS to obtain maximum Stokes energy as a function of the number of passes. The meanings of the different lines are the same as those in Fig. 4

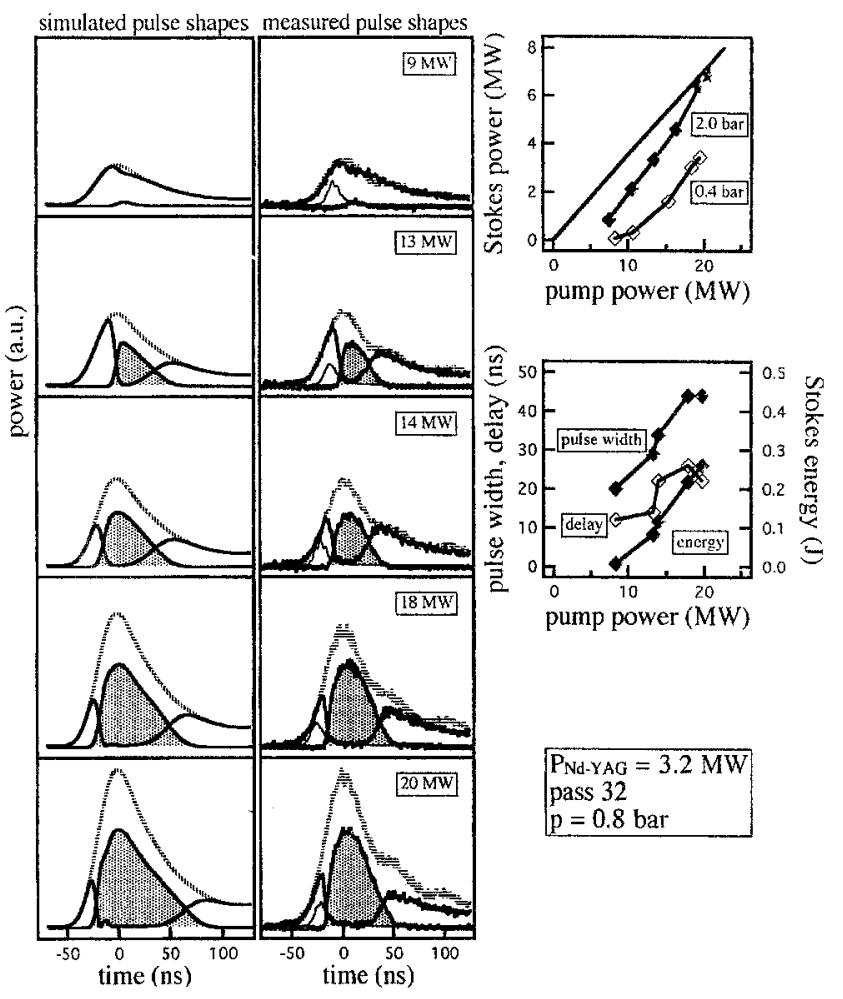

Fig. 6. Stokes energy, pulse width, power, and the delay in seeded SRS to obtain maximum Stokes energy as a function of the pump power. The meanings of the different lines are the same as those in Fig. 4

which maximum energy was scattered occurs for pump powers which cause full depletion. For low pump powers which approach threshold, the delay had to be decreased.

The pressure dependence is presented in Fig. 7. The delay between the two pump pulses is largest at gas pressures of approximately $0.6-0.8$ bar. In this range, the highest Stokes energy and pulse width are found, while the Stokes power saturates. For higher gas pressures, pump depletion is full, while the Stokes-pulse width decreases.

Summarizing these results, the measured input- and output-pulse shapes show that the Stokes energy is generated in a pulse much wider than the Nd:YAG pulse. The width of the Stokes pulse depends among others on gas pressure and on the position of the Nd:YAG pump pulse.

\section{Numerical calculations}

The pulse shapes of the Stokes and depleted pump pulses were simulated in calculations based on the coupled equations for the pump and Stokes waves and the Raman polarizability for transient SRS, which were derived from semiclassical SRS theory $[8,9]$ :

$$
\begin{gathered}
\frac{\partial}{\partial z^{\prime}} E_{\mathrm{p}}\left(z^{\prime}, t^{\prime}\right)=-K_{\mathrm{p}} E_{\mathrm{S}} Q, \\
\frac{\partial}{\partial z^{\prime}} E_{\mathrm{S}}\left(z^{\prime}, t^{\prime}\right)=K_{\mathrm{S}} E_{\mathrm{p}} Q^{*},
\end{gathered}
$$

$\left(\frac{\partial}{\partial t^{\prime}}+\Gamma\right) Q^{*}=E_{\mathrm{p}}^{*} E_{\mathrm{S}}$ 


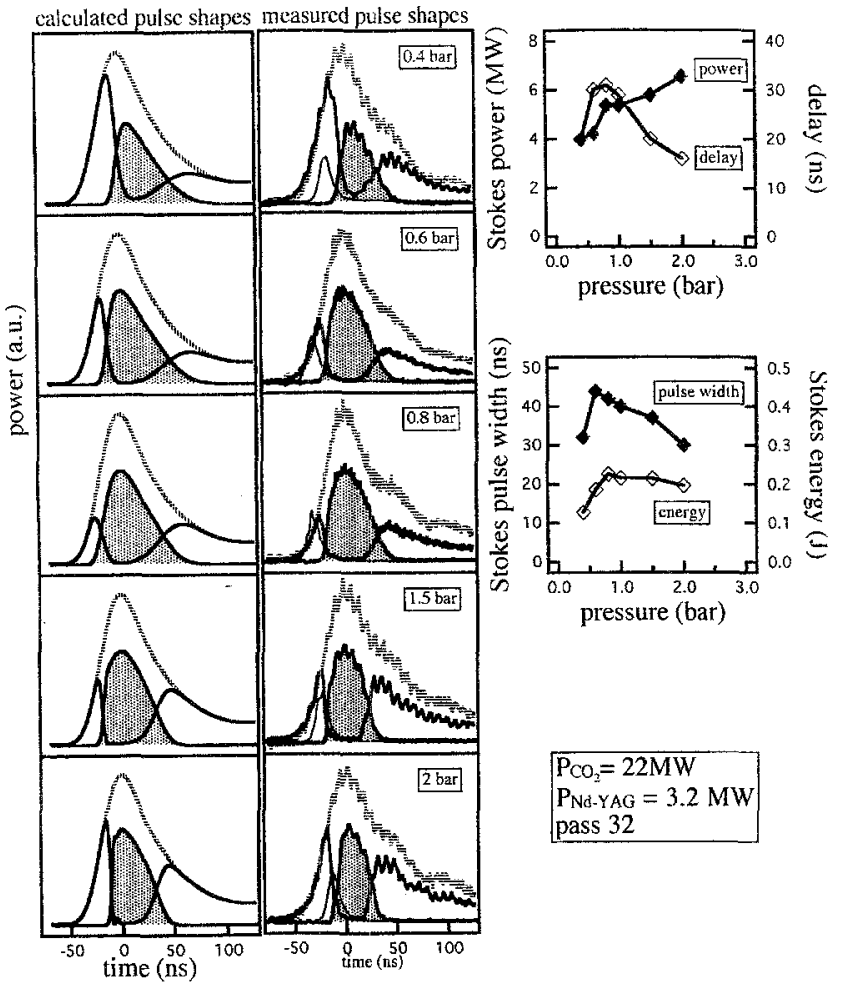

Fig. 7. Stokes energy, pulse width, power, and the delay in seeded SRS to obtain maximum Stokes energy as a function of pressure. The meanings of the different lines are the same as those in Fig. 4

Here, $K_{\mathrm{p}}$ and $K_{\mathrm{S}}$ are coupling constants for which the ratio $K_{\mathrm{p}} / K_{\mathrm{S}}$ equals $\omega_{\mathrm{p}} / \omega_{\mathrm{S}}, E_{\mathrm{p}}$ and $E_{\mathrm{S}}$ are the electric-field amplitudes of the pump and Stokes waves, respectively, and $Q$ the amplitude of the material excitation which is proportional to the Raman polarizability; $z^{\prime}$ and $t^{\prime}$ are the space and time coordinates, respectively, in the moving frame of the optical pulses. Medium dispersion is assumed to be negligible. The input pump-pulse shape was taken from experimental data, where the small modulations due to mode beating were smoothed out. The input Stokespulse shape was Gaussian with a pulse width of $9 \mathrm{~ns}$ at half maximum. Its peak amplitude was taken proportional to the pump amplitude in the pump pulse section with which it overlapped to simulate the dependence on pump power in FWM. Furthermore, a Stokes-noise power level was added. In the calculations relative values for the Stokes-noise power and pump-power levels were used. The values for $K_{\mathrm{p}}$ and $K_{\mathrm{S}}$ were chosen such as to produce efficient conversion, while $K_{\mathrm{p}} / K_{\mathrm{S}}$ was equal to $\omega_{\mathrm{p}} / \omega_{\mathrm{S}}$. Therefore, the powers in the output pulse shapes are given in arbitrary units. The interaction length $L$ was chosen 10 times longer than the full width $\Delta t$ of the pump pulse at half maximum, i.e., $L=10 \mathrm{c} \Delta t$ where $c$ is the light velocity. Each of the Figs. 4, 6, and 7 shows the output pulse shapes. In general, the onset as well as the asymmetry of the pump depletion is well reproduced.

Initially, the short seed pulse is amplified by SRS of the small overlapping section of the pump pulse. In the experiments, this pump-pulse section is determined by the delay between the $\mathrm{Nd}$ :YAG and $\mathrm{CO}_{2}$ pump pulses. Once energy conversion in this pump-pulse section has started, a large
Raman polarizability can be established by the beat of the pump wave and the growing Stokes wave. Due to its finite dephasing time, a decaying polarizability remains when the Stokes pulse and the overlapping section of the pump pulse propagate. This polarizability induces SRS of the following pump-pulse sections more efficiently. The polarization generated at the Stokes frequency then grows and may radiate at measurable powers causing these pump-pulse sections to deplete. At the same time, the amplified Stokes pulse beats with the copropagating pump-pulse section to enforce the Raman polarizability. Even for dephasing times which are smaller than the pump-pulse width by one order of magnitude, the polarizability is sustained up to the tail of the pump pulse. This results in long Stokes pulses and asymmetric pump depletion.

The dephasing time is determined by the gas pressure and influences the build-up time of the polarization at the Stokes frequency. Consequently, it influences the delay between the Nd:YAG and $\mathrm{CO}_{2}$ pump pulses at which maximum energy is scattered from the $\mathrm{CO}_{2}$ pump pulse to the Stokes pulse. The shore dephasing time at high gas pressures implies a fast growing or decaying polarization. For maximum energy conversion, the Stokes seed must then be generated close to the pump-pulse peak. Experimentally, the $\mathrm{CO}_{2}$ pump pulse must follow the Nd:YAG pump pulse with a small delay (Fig. 7). The longer dephasing time at lower gas pressures allows a wider Stokes pulse but the polarization needs a longer time to build up. Hence, the delay between the Nd:YAG pump pulse and the trailing $\mathrm{CO}_{2}$ pump pulse must be increased. The widest Stokes pulse with the highest energy is obtained with a dephasing time which corresponds to a gas pressure of about 0.8 bar. The occurrence of an optimum pressure is a result of the increase of the Raman threshold at the low-pressure side and the reduced dephasing time of the polarizability which sustains the Raman scattering at the high-pressure side. At this optimum gas pressure, the pulse-shape behavior is shown in Fig. 4. In the first frame of the series, the Stokes pulse is found about 70 ns (i.e., approximately 25 dephasing times) after the Nd:YAG pulse. Apparently, such time is necessary for the polarization at the Stokes frequency to become large enough to radiate at the power level just above threshold. This particular frame is illustrative for SRS which is induced solely by the decaying polarizability generated by SRS of the preceding Nd:YAG pump pulse [10] instead of a seed generated by FWM. Because the $\mathrm{Nd}: \mathrm{YAG}$ and $\mathrm{CO}_{2}$ pump pulses do not overlap, no FWM seed at the Stokes frequency is likely to be generated. At the lowest pressure of 0.4 bar in the experiments (Fig. 7), a maximum Stokes power was found for a Nd:YAG pump pulse which preceded the $\mathrm{CO}_{2}$ pump pulse by $18 \mathrm{~ns}$. Because of the long dephasing time, the scattering is strongly transient. The Stokes pulse developed to powers above threshold $40 \mathrm{~ns}$ after the Nd-YAG pump pulse was applied and depleted the $\mathrm{CO}_{2}$ pump pulse in the trailing edge, causing the asymmetry of the depletion. For longer dephasing times, the polarization would grow even slower and would be unable to cause pump depletion within the pulse duration. Thus, threshold for seeded SRS would not have been obtained at lower gas pressures. 


\section{Summary and conclusions}

Experiments and simulations on SRS, which is induced by a seed pulse at the Stokes frequency with a pulse duration much shorter than the pump pulse, show high conversion efficiencies for pump powers well below threshold for unseeded SRS. The generated Stokes pulse is substantially longer than the seed pulse or the medium dephasing time. The shapes of the Stokes and depleted pump pulses which are generated in seeded SRS are well described by the set of coupled equations for SRS derived from semiclassical theory. Therefore, seeding only initiates efficient scattering, while energy conversion is by pure Raman scattering.

Acknowledgements. The authors would like to thank H.T.M. Prins, G.J.M. Oude Mijers and A.F. Nieuwenhuis for their technical assistance. Partial financial support by FOM (Foundation for Fundamental Research on Matter) of one of the authors, R.J. Heeman, is gratefully acknowledged.

\section{References}

1. K. Drühl, R.G. Wenzel, J.L. Carlsten: Phys. Rev. Lett. 51, 1171 (1983)

2. R.A.J. Keyser, J.R. Lombardi, K.D. van den Hout, B.C. Sanctuary, H.F.P. Knaap: Physica 76, 585 (1974)

3. R.J. Heeman, H.P. Godfried: IEEE J. QE-31 (2) (1995)

4. W.R. Trutna, R.L. Byer: Appl. Opt. 19, 301 (1980)

5. G.C. Bjorklund: IEEE. J. QE-11, 287 (1975)

6. R. Holmes, A. Flusberg: Phys. Rev. A 37, 1588 (1987)

7. R.J. Heeman, H.P. Godfried: IEEE J. Quantum Electron. (in press)

8. R.L. Carman, F. Shimizu, C.S. Wang, N. Bloembergen: Phys. Rev. A 2, 60 (1970)

9. Y.R. Shen: The Principles of Nonlinear Optics (Wiley, New York 1984) pp. 176-181

D.L. Mills: Nonlinear Optics (Springer, Berlin, Heidelberg 1991)

10. L. Marshal, J. Piper: Opt. Lett. 15, 1345 (1990); IEEE J. QE-29, $515(1993)$ 